\title{
Burn Cases, Their Management and Complications: A Review
}

\author{
${ }^{*}$ Rizwan Ali Masood ${ }^{1}$, Zafeer Naeem Wain"1, ${ }^{1}$, Rehan Tariq ${ }^{1}$, Muhammad Asis ullah ${ }^{1,2}$, Irfan Bashir ${ }^{1,2}$ \\ ${ }^{1}$ Faculty of Pharmacy, University of Central Punjab, Lahore, Pakistan \\ ${ }^{2}$ Foundation for Young Researchers, Lahore, Pakistan
}

\begin{abstract}
Skin being the primary barrier to infection can be damaged by burn injury. Burn injury may lead to distributive, hypovolemic and cardiogenic shock. A burn victim may experience several extremely mortal complications i.e. local and systemic. When the injury exceeds 25 to $30 \%$ total body surface area (TBSA) there will be the chances of generalized edema in non-injured tissues. Fluid resuscitation is very effective method in the management of major burn. According to the expert opinion, the fluid resuscitation should be started in adults with $15 \%$ and children with $10 \%$ burns. In this review article, it has been concluded that opioids, anticonvulsants, antidepressants, benzodiazepines and ketamine are the most commonly used drugs in the management of burn pain. Silver compound or its salts mainly Silver sulfadiazine is an important remedy in topical treatment. Sepsis is the main cause of death in burn victims.
\end{abstract}

Key Words: Cardiogenic, Fluid shift, Hypovolemic, Hypothermia, Total body surface area

\section{INTRODUCTION}

Injuries to the skin and organic tissues due to contact with chemicals, electricity, friction, radiation and radioactivity are known as burn. Burn injury happens once some or all the cells within the skin or tissues area unit is broken by hot flame, solids and liquids (Peden, 2008). Skin being the primary barrier to infection can be damaged by burn injury (Lee et al., 2014).

Burn injury leads to distributive, hypovolemic and cardiogenic shock. Due to increased capillary permeability and fluid shift, the intravascular volume becomes depleted. The intravascular volume becomes depleted primarily owing to accumulated capillary permeableness and fluid shifts. If more than $30 \%$ total body surface (TBSA) is involved, partial compensation can be obtained by fulfilling the fluid requirement of body and cause a recession in sodium ATPase level and its action, leading to desorption of trans-membrane ionic gradient of cell which may prolong for couple of days (Snell et al., 2013).

In case of micro-vascular injury different inflammatory mediators like prostaglandins, vasoactive amines, bradykinin, and platelet activating product, leukotrienes and histamine are released followed by loss of proteins in the interstitium (Ahrns et al., 2004).

When the injury exceeds 25 to $30 \%$ total body surface area (TBSA), there will be the chances of generalized edema in non-injured tissues. Followed by the major burn injury, there will be continuous loss of plasma into burned tissue can last for first $48 \mathrm{~h}$ or longer. Because of intravascular fluid into burned areas and edema formation (in nonburned sites) the burn shock may occur which is characterized with impaired tissue and organ perfusion (Bittner et al., 2015).
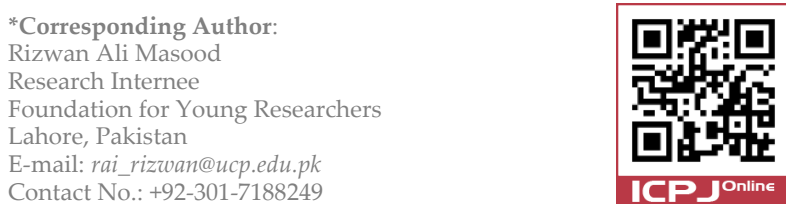

CLASSIFICATION OF BURN

- $\quad$ First-degree burns: The epidermis is involved in first degree burns. Which are like sun burn, erythematous, sore, and coarse. The minor thermal injury or exposure to severe ultraviolet radiation may cause first degree burns. Their healing time is 5 to 10 days (Emillia et al., 2012).

- Second-degree burns: It is further divided into two categories:

- A superficial partial thickness burn: They usually invade into the superficial papillary dermis. They are characterized with reddish blisters. When pressure is applied, the blisters may shrink and their healing time is $2-3$ weeks.

- Deep partial thickness burns: They penetrate the reticular dermis and are yellow or white in color, rough in nature and are very painful. They require more than 3 weeks for complete healing (Jimmy Toussaint and Adam J. Singer, 2014).

- Third-degree burns: They damage both inner and outer layers of the skin, that's why this is the most severe type of burns. They are white in color and usually non-achy. Only a small number of such type of burns heal own their own which is a long-time process (Torpy et al., 2009).

- Fourth degree burn: They invade into the harmed muscles, ligaments, tendons, nerves, blood vessels, and bones, through the skin. Severe medical emergency treatment is required for this kind of burns. They are black and scorched (Vadukul, 2012).

\section{DETERMINATION OF BURN DEPTH}

The methods used for determining burn depths are vital dyes, tissue biopsies and ultrasound. These methods can detect damaged collagen or dead cells. To evaluate changes in blood flow, fluorescein perfusion fluoroscopy, laser doppler flowmetry and thermography methods are used. Burn depth can also be observed by nuclear magnetic resonance (Jimmy Toussaint and Adam J. Singer, 2014). 


\section{TYPES OF BURN}

Following are the different type of burns.

Thermal burn: They are caused by flashed light, flame, blazing, or contact with a hot surface.

- Explosions of flammable liquids, natural gas, propane, gasoline results into flash burns. Intense heating is involved in flash burns that lasts for short period.

- $\quad$ Flame burns are usually caused by prolonged exposure to intense heat, frequently associated with clothing ignited by stoves and heaters, improper use of flammable liquids, automobile accidents and house fires.

- Scalds involve hot liquids like water, oil, grease or tar. A deep burn can be caused by water at 140 degrees (F) in 3 seconds, but same injury will be resulted in just one second at 156 degrees $(F)$.

- Contact burns are caused by hot coals, plastics, metals or glass. They may be painful and deep (Schmid, 2015).

Chemical burn: They are caused by exposure to reactive chemical substances such as strong acids or alkalis (Gnaneswaran et al., 2015).

Electrical burn: Passage of electrical current from an electrical outlet or appliance through the body may results into the electrical burn (Buja et al., 2010).

Radiological burns: Alpha, beta or gamma radiations are responsible for radiological burn. To stop the injury process there is a need of decontamination procedure for the people exposed to these types of radiation (Schmid, 2015).

\section{COMPLICATIONS}

The complications of the injury vary up to a great extent depending on the affected location, tissue and the degree of severity. Scar formation and deformity due to burns cause psychological discomfort in patients (Perera et al., 2015)

Systemic and local both type of complications is caused by burn. Fluid loss and breakdown of skin integrity is major contributor of systemic complications. Contractures, scarring and eschars are the local complications of burn injury (Rowan et al., 2015).

\section{Systemic}

The risk of developing systemic complications is directly proportional to the total body surface area (TBSA) involved in burn injury. Following are the risk factors of severe systemic complications and mortality.

- $\quad$ Burns of more than $40 \%$ of TBSA

- Age of greater than 60 year or less than 2 year

- Presence of concurrent smoke inhalation or major trauma

Hypovolemia and infection are the most common systemic complications (Singh et al., 2007).

\section{Local}

Eschar "rigidness and death of tissue caused by deep burns". Respiration can be compromise by an eschar around the thorax and viability of limbs and digits is endangered by ischemia (Rowan et al., 2015).

Scarring and contractures due to healing of deep burns; contracture deformities can appear at the joints depending on the extent of the scar.

\section{Infection in burns}

Bacterial growth on damaged skin along with immunosuppression causes wound infection, invasive sepsis and may lead to death. The mortality rate caused by hypovolemia and hyperosmolar shock is reduced with the increase of resuscitation procedures in burn patients (Lee et al., 2014).

\section{MANAGEMENT OF BURN}

\section{First Aid Management}

- $\quad$ The principles of first aid are

- to stop the burning process

- to cool the burn wound

- $\quad$ Lowering the temperature of the burnt surface acts as an effective pain killer. It is effective only if done within 3 hours of the injury.

- $\quad$ Prolonged cooling may lead to hypothermia in small children.

- Avoid the use of ice water or ice, it may alleviate the risk of hypothermia as well as depth of the tissue injury (Hasselt, 2008).

\section{Fluid resuscitation}

- In the managements of major burns effective fluid resuscitation is very important. Intravenous fluids should be given "on scene," in case of $25 \%$ burn. After more than 2 attempts at cannulation transfer should not be delayed (Enoch et al., 2009).

- To prevent extension of the thermal necrosis, it is necessary to maintain vital organ perfusion and tissue perfusion around the burn. According to the expert opinion the fluid resuscitation should be started in adults with $15 \%$ burns and children with $10 \%$ burns. There will be minor chances of sepsis, renal failure and death in children who received fluid resuscitation within 2 hours (Haberal et al.,2010).

- There is a great variation in the preferd resuscitation fluid. Crystalloid Hartmann's solution is the most common resuscitation fluid which cures hypovolaemia and deficiency of extracellular sodium. Normal saline should be avoided as it may result hyperchloraemic metabolic acidosis (Enoch et al,.2009).

\section{Pain management in burn}

The direct stimulation and injury of the nociceptors present in the dermis and epidermis may results into the pain. As a result of which $C$ fibers and A-delta send nerve impulses to the spinal cord dorsal horn. The descending influences and peripheral stimuli from the brain cause modulation of impulse magnitude (Castro et al., 2013).

According to the nature and intensity of pain in burn patients the most effective treatment is drug administration. In pain management following drugs are used: (Richardson and Mustard, 2009).

- Opioids; in pain management, they play a vital role. Non-steroidal anti-inflammatory drugs (NSAIDs) \& anti-inflammatory drugs (paracetamol and dipyrone), reduce the adverse effects of opioids.

- Anticonvulsants: For treating neuropathic pain in burn patients, gabapentin and pregabalin are often used.

- Antidepressants also play an important role in the cure of pain in burn patients.

- In burn patients, for conscious sedation ketamine is being used during dressing changes.

- $\quad$ Benzodiazepines; In case of exacerbated pain caused by anxiety disorders, the anxiolytics are used along with analgesic (Castro et al., 2013). 


\section{Topical therapies}

Silver compounds or salts in topical burn therapy play a key role and reducing the rate of sepsis, burn wound and death. Silver sulfadiazine is an important remedy in topical treatment because of its successful infection control and minimizing the side effects (Hyland et al., 2015).

Mafenide acetate (Sulfamylon) was used as an alternative of silver compound solutions but because of carbonic anhydrase inhibitory effects (which can lead to systemic acidosis), its use was discontinued but still it is used to invasive wound infections. Silver nitrate is also one of the common silver base therapy. The infections such as Pseudomonas aeroginosa infections were successfully treated with silver based topical treatment (Dai et al., 2010).

The use of dressings and gels comprising of glycose, minoglycan and chitin is becoming evident now-a-days. This prevents early extension of burn injury. They usually have antimicrobial characteristics which are responsible for the angiogenesis and fibroblast proliferation which may lead to healing of the burn wounds. The use of carbon fiber in dressings increases the absorptive capacity of the dressing that reduce bacterial growth, promote healing and reduce inflammation (Lee et al., 2014).

\section{Non-Pharmacological therapies}

In ancient times, for the management of burn honey has been used. Maternal milk, mixture of gums and goat's hair had been used by Egyptians for the treatment of burns. Since the 20th century various local medications have been used. Due to its hepatotoxicity, the use of tannic acid spray was rejected in 1942 (Justin-Temu et al., 2008).

In the management of pain and anxiety in burn patient non-pharmacological therapy is complementary. The development of anxiety and the cycles of pain can be prevented by initiating non-pharmacological therapy as early as possible. The way should be versatile, involving pain specialists, psychotherapists and physiotherapists. During rehabilitation pain and anxiety can be relieved by using Psychological techniques such as cognitive behavioral therapy, relaxation and distraction (Castro et al., 2013).

\section{CONCLUSION}

Burn management depends upon its severity. Opioids, anticonvulsants, antidepressants, benzodiazepines and ketamine are the most commonly drugs used in the management of pain. Silver compound or its salts mainly Silver sulfadiazine is an important remedy in topical treatment. The use of honey and milk were also most common in ancient times. Systemic and local both type of complications is caused by burn. Fluid loss and breakdown of skin integrity is major contributor of systemic complications. Contractures, scarring and eschars are the local complications of burn injury.

\section{CORRESPONDING AUTHOR PROFILE}

Rizwan Ali Masood is a fourth year Pharm.D student in Faculty of Pharmacy, University of Central Punjab Lahore, Pakistan. He has keen interest in research topics related to community health issues and is actively involved in various Public Health Awareness Campaigns being conducted through platform of different NGOs.

\section{REFERENCES}

Ahrns, K. S. (2004). Trends in burn resuscitation: shifting the focus from fluids to adequate endpoint monitoring, edema control, and adjuvan therapies. Critical care nursing clinics of North America, 16(1), 75-98. [DOI]

Bittner, E. A., Shank, E., Woodson, L., \& Martyn, J. J. (2015). Acute and perioperative care of the burn-injured patient. The Journal of the American Society of Anesthesiologists, 122(2), 448-464. [DOI]

Buja, Z., Arifi, H., \& Hoxha, E. (2010). Electrical burn injuries. An eightyear review. Ann Burns Fire Disasters,23(1), 4-7.

Castro, R. J. A. D., Leal, P. C., \& Sakata, R. K. (2013). Pain management in burn patients. Revista Brasileira de Anestesiologia, 63(1), 154-158. [DOI]

Dai, T., Huang, Y. Y., K Sharma, S., T Hashmi, J., B Kurup, D., \& R Hamblin, M. (2010). Topical antimicrobials for burn wound infections. Recent patents on anti-infective drug discovery, 5(2), 124-151. [DOI]

Enoch, S., Roshan, A., \& Shah, M. (2009). Emergency and early management of burns and scalds. BMJ, 338. [DOI]

Gnaneswaran, N., Perera, E., Perera, M., \& Sawhney, R. (2015). Cutaneous chemical burns: assessment and early management. Australian family physician, 44(3), 135.

Haberal, M., Abali, A. E. S., \& Karakayali, H. (2010). Fluid management in major burn injuries. Indian Journal of Plastic Surgery, 43(3), 29. [DOI]

Hasselt (2008) Burn Manual, A manual for health workers. Blantyre Malawai: Nederlandse Brandwonden Stichting.

Hyland, E. J., Connolly, S. M., Fox, J. A., \& Harvey, J. G. (2015). Minor burn management: potions and lotions. Australian prescriber, 38(4), 124. [DOI]

Justin-Temu, M., Rimoy, G., Premji, Z., \& Matemu, G. (2008). Causes, magnitude and management of burns in under-fives in district hospitals in Dar es Salaam, Tanzania. East African journal of public health, 5(1), 3842. [DOI]

Lee, K. C., Joory, K., \& Moiemen, N. S. (2015). History of burns: The past, present and the future. Burns \& Trauma, 2(4), 169.

M.M.Peden.(2008).World Report On Child Injury Prevention.Geneve,World Health Organization and International Society for Burn Injuries.

Perera, M. M. N., Nanayakkarawasam, P. P., \& Katulanda, P. (2015). Effects of burn on the mobility of upper limb/s, functions of hand/s \& activities of daily living. International Journal of Physiotherapy and Research, 3(1), 832-8. [DOI]

Richardson, P., \& Mustard, L. (2009). The management of pain in the burns unit. Burns, 35(7), 921-936. [DOI]

Rowan, M. P., Cancio, L. C., Elster, E. A., Burmeister, D. M., Rose, L. F., Natesan, S., ... \& Chung, K. K. (2015). Burn wound healing and treatment: review and advancements. Critical Care, 19(1), 1. [DOI]

Singh, V., Devgan, L., Bhat, S., \& Milner, S. M. (2007). The pathogenesis of burn wound conversion. Annals of plastic surgery, 59(1), 109-115. [DOI]

Snell, J. A., Loh, N. H. W., Mahambrey, T., \& Shokrollahi, K. (2013). Clinical review: the critical care management of the burn patient. Critical Care, 17(5), 1. [DOI]

Torpy, J. M., Lynm, C., \& Glass, R. M. (2009). Burn injuries. JAMA, 302(16), 1828-1828. [DOI]

Toussaint, J., \& Singer, A. J. (2014). The evaluation and management of thermal injuries: 2014 update. Clinical and Experimental Emergency Medicine, 1(1), 8-18. [DOI]

Vadukul, K (2012) Burn Injuries Require Quick Identification, Treatment \& Transport. Journal of Emergency Medical Services, 37(12). 